\title{
AEROTHERMAL AND FLIGHT MECHANIC CONSIDERATIONS BY DEVELOPMENT OF SMALL LAUNCHERS FOR LOW ORBIT PAYLOADS STARTED FROM LORENTZ RAIL ACCELERATOR
}

\author{
O. Božić, T. Eggers, and S. Wiggen \\ DLR - Institute for Aerodynamics and Flow Technology \\ Lilienthalplatz 7, Braunschweig 38108, Germany
}

The injection of small payloads in Low Earth Orbit (LEO) by means of propelled launchers starting from a Lorentz Rail Accelerator (LRA) is a concept that may enable the access to space at extremely low cost. A propelled launcher is required since today a LRA is only able to launch a total mass of a few kilograms with a velocity up to $4.4 \mathrm{~km} / \mathrm{s}$ but LEOpayloads require approximately $10 \mathrm{~km} / \mathrm{s}$ at higher launch mass. Velocity difference must be assured with another propulsion system. Furthermore and independent of the type of selected propulsion, such solution has serious consequences on launcher design. Reasons are, e.g., the harsh mechanical loads like high acceleration on the LRA ramp, high deceleration due to pressure drag, unsteady phenomena during the transition from the LRA ramp into the free atmosphere and also due to extreme thermal loads in the first $30 \mathrm{~s}$ of flight. The study presents a conceptual design of a nominal payload of $3 \mathrm{~kg}$, including dimensions, mass- and velocitybudget estimations. In the focus of the analysis are several concepts for the thermal protection of critical system like the nose cap, the front part of the fuselage which houses a hybrid kick-off engine, flares, and the attitude control engines. Additionally, the potential of plug nozzles in comparison to classical Laval nozzles as well as trajectory calculations are discussed. They underline that an elliptical orbit between 300 and $400 \mathrm{~km}$ is possible.

\section{NOMENCLATURE}

$$
\begin{array}{ll}
D_{C} & \text { diameter of the cylindrical body }(\mathrm{m}) \\
H_{L, \mathrm{SP}} & \text { stagnation point heat flux }\left(\mathrm{W} / \mathrm{m}^{2}\right)
\end{array}
$$

This is an Open Access article distributed under the terms of the Creative Commons Attribution-Noncommercial License 3.0, which permits unrestricted use, distribution, and reproduction in any noncommercial medium, provided the original work is properly cited. 


$\begin{array}{ll}I_{\mathrm{sp}} & \text { specific impulse }(\mathrm{s}) \\ L_{\mathrm{nc}} & \text { nose cap length }(\mathrm{m}) \\ L^{\prime} & \text { inductance gradient }(\mathrm{H} / \mathrm{m}) \\ \mathrm{Ma} & \text { Mach number } \\ m_{t} & \text { launch package mass }(\mathrm{kg}) \\ R & \text { nose radius }(\mathrm{m}) \\ R_{c} & \text { radius of the cylindrical body }(\mathrm{m}) \\ T_{\mathrm{BW}} & \text { back wall temperature }(\mathrm{K}) \\ T_{W, \mathrm{SP}} & \text { stagnation point temperature }(\mathrm{K}) \\ V_{0} & \text { initial } / \text { start velocity }(\mathrm{m} / \mathrm{s}) \\ w_{t} & \text { weight }(\mathrm{kg}) \\ \gamma & \text { flight path angle }\left(^{\circ}\right)\end{array}$

\section{ABBREVIATIONS}

$\begin{array}{ll}\text { ADCS } & \text { Attitude Determination and Control System } \\ \text { CFRP } & \text { Carbon Fibre Reinforced Plastic } \\ \text { DES } & \text { Distributed Energy storage System } \\ \text { EML } & \text { Electro-Magnetic Launcher } \\ \text { HRE } & \text { Hybrid Rocket Engine } \\ \text { HTP } & \text { High Test Peroxide (more than } 90 \% \mathrm{H}_{2} \mathrm{O}_{2} \text { ) } \\ \text { LEO } & \text { Low Earth Orbit } \\ \text { LRA } & \text { Lorentz Rail Accelerator } \\ \text { MBA } & \text { Monolitic Braided Ablative } \\ \text { O/F } & \text { Oxidizer/Fuel ratio } \\ \text { RANS } & \text { Reynolds-Averaged Navier-Stokes } \\ \text { RCS } & \text { Reaction Control System } \\ \text { SMES } & \text { Superconductive Magnetic Energy Storage } \\ \text { SP } & \text { Stagnation Point } \\ \text { TPS } & \text { Thermal Protection System } \\ \text { UHTC } & \text { Ultra High Temperature Ceramics }\end{array}$

\section{INTRODUCTION}

In the last time, a number of the third world countries tried to develop aerospace capacities and launch own small satellites. The trend to miniaturization of satellites increases due to the progress in mechatronic and automatization. Meanwhile, a number of private companies, universities, and nongovernmental institutions show interest for small orbital platforms for different purposes. This is a growing market with future potential which covers pico-, nano- and microsatellite assortment. One important market segment is the nanosatellite (mass range 
1-10 kg) production. Possible application areas of such satellites are: testing and validation of components, technologies and concepts for space applications, execution of complex scientific experiments, local and regional communications, inspections and service missions, remote sensing for miscellaneous applications. In the near future, it is expected to see small satellites in formation flight for radioastronomic research and communication grids. One study carried by DLR [1] has shown that the demand on low cost and flexible orbital launch for small payloads is growing and this market segment is the primary target for the proposed electromagnetic launcher. The maturity of the electromagnetic LRA technology has reached a level that makes it a potential candidate for such tasks. The high performance expected concerning velocity, efficiency, cost, and repetitions rate make this system attractive for space applications with the aim of accelerating lightweight payloads into LEO.

Mission requirement is to deliver the picosatellites up to $3 \mathrm{~kg}$ mass in an equatorial LEO. Two possible scenarios are intended. The first mission scenario foresees the insertion of picosatellite in a medium light elliptical orbit, established within thermosphere $(90-500 \mathrm{~km})$. One reasonable initial orbit should be between 300 and $400 \mathrm{~km}$. Final orbit tuning can be realized with ADCS propulsion of the satellite itself. The second scenario foresees the injection of a payload into a high elliptical transfer orbit within exosphere (500-2000 km). For both scenarios, the main properties of the LRA launcher and rocket payload carrier have to be determined. A promising solution is a combined application of LRA and a chemically propelled carrier. This paper attempts to define limitations and possible "show-stoppers" connected with rocket assisted LRA launch to LEO.

\section{LORENTZ RAIL CATAPULT PERFORMANCES}

In the past, two research institutions in the U.S. carried, primarily for military purposes, the major part of investigations on electromagnetic rail accelerators (LRA): "Green Farm" by San Diego in California (DARPA, 1992) and the Institute of Advanced Technology at the University of Texas, Austin - Center of Electromagnetics (IAT-UT/CEMT). The power device on the "Green Farm" facility with a condenser bank was capable of delivering the input energy of 32 MJ. The 9-meter long LRA with plasma armature and a bore of $90 \mathrm{~mm}$ was tested during 1988 and 1998, performing 254 shots. Highlights of these tests were vehicle shots with a mass of $m=0.64 \mathrm{~kg}$ (the corresponding kinetic energy was $5.95 \mathrm{MJ}$ and start velocity $V_{0}=4.3 \mathrm{~km} / \mathrm{s}$ ) and a mass of $m=1.13 \mathrm{~kg}$ (the corresponding kinetic energy was $7.0 \mathrm{MJ}$ and $V_{0}=3.5 \mathrm{~km} / \mathrm{s}$ ). The IAT-UT/CEMT also installed a 90-millimeter LRA facility capable of launching a vehicle with kinetic energy $P_{e}=9 \mathrm{MJ}$. As a power source, homopolar generators were used instead of capacitors [2]. Since 2003, they develop a LRA launcher with a bore 


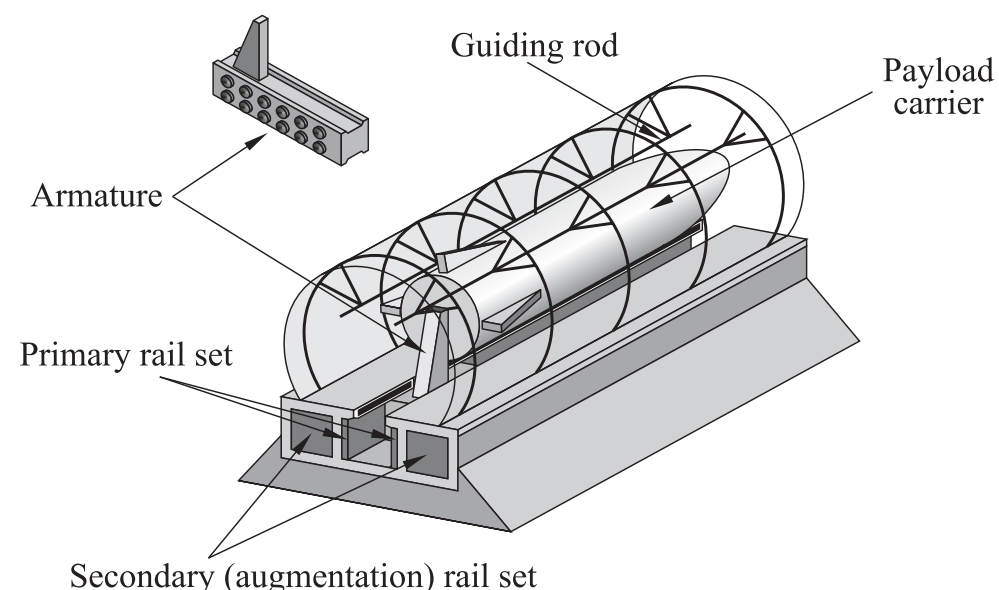

Figure 1 Displaced position of rocket carrier and armature on the LRA ramp segment

of $155 \mathrm{~mm}$ and a length of $10 \mathrm{~m}$ [3]. The leading European Institution is the French-German Institute (ISL) in Saint-Louis; France which has developed the LRA "Pegasus." This 6-meter long accelerator with an input energy of $32 \mathrm{MJ}$ is capable of launching payloads of $0.5 \mathrm{~kg}$ up to $2.1 \mathrm{~km} / \mathrm{s}$ [4]. The last achievement in this field of science is a test with a LRA carried out at the Naval Surface Warfare Center, Dahlgreen, Virginia, USA (NSWCDD) in January 2008. A 3.2kilogram vehicle was launched with a start velocity of $2.5 \mathrm{~km} / \mathrm{s}$. The kinetic energy of this vehicle was $10.8 \mathrm{MJ}$ which is a new record for launched packages. The corresponding input energy for the LRA was 32 MJ. The final goal planed for the year of 2020 is to build a system with kinetic energy of 64 MJ being capable of launching a guided vehicle of 15-kilogram mass [3].

Within a former DLR feasibility study,

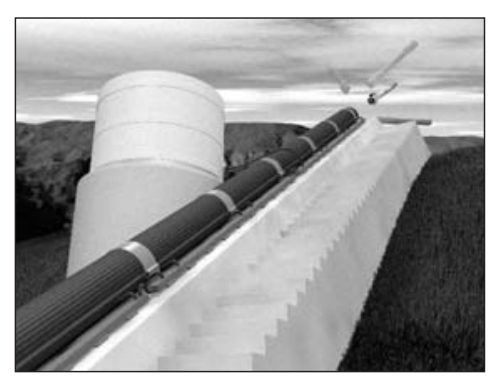

Figure 2 Display of Lorentz accelerator catapult ramp the different LRA concepts were analyzed.

The LRA concept selected here is a segmented and parallel augmented ramp accelerator. More details concerning the operation principle, the present state of development, power feeding, and technical limitations by application of solid, plasma, and hybrid armatures are given in [4-7]. For launch packages with a diameter larger than $200 \mathrm{~mm}$, LRA should be of ramp type (Figs. 1 and 2). 
Table 1 Properties of LRA ramp system

\begin{tabular}{lcc}
\hline Lorentz rail accelerator (ramp type) & Scenario 1 & Scenario 2 \\
\hline Start velocity $V_{0}, \mathrm{~m} / \mathrm{s}$ & 3550 & 4400 \\
Maximum/medium initial acceleration $a_{M}, g$ & $3300 / 2823$ & $3300 / 2823$ \\
Gradient of inductance $L^{\prime}, \mu \mathrm{H} / \mathrm{m}$ & 0.5 & 0.5 \\
Maximal armature current $I, \mathrm{kA}$ & 6000 & 6000 \\
Mach number at start (ramp end) Ma & 10.45 & 12.96 \\
Number of rail ramp segments $N$ & 38 & 56 \\
Total rail length $L, \mathrm{~m}$ & 228 & 350 \\
Kinetic energy at start $E_{k}, \mathrm{GJ}$ & 4.1 & 6.3 \\
Input energy - facility $E_{o}, \mathrm{GJ}$ & 13.65 & 20.97 \\
Launch package mass $m_{\mathrm{LP}}, \mathrm{kg}$ & 651 & 651 \\
Residence time of payload carrier $t$ & & \\
on the ramp during launch, ms & 128 & 159 \\
\hline
\end{tabular}

Here, the armature/sabot and the rocket holder are connected with a wedge which transfers the momentum from the armature/holder and pushes the projectile. If the tube (bore) diameter is between 50 and $200 \mathrm{~mm}$, the LRA can achieve its maximum electrical efficiency. Concurrently, this solution does not limit the projectile span due to the flare/fins necessary for aerodynamic stabilization. To obtain the required launch velocity (see Table 1 for details), the total length of the rails is between 228 and $350 \mathrm{~m}$. A classical segmented LRA, for the same armature current and inductivity gradient needs a double ramp length to achieve the same velocity.

The length limitation imposed by the rail resistance and rail inductance can be circumvented by subdividing the shorter LRA into 38 and longer LRA into 56 segments (Table 1). Each segment can be fed by an independent local energy source. This modular design could enable building a section from the available inexpensive materials and assembling it in place. For a segmented LRA with numerous sections, the problem of switching a high number of power units must be solved. In such LRA construction, the only moving element is the armature with brushes.

The LRA ramp is installed inside a tube with semicircled intersection. The air inside the tube is evacuated (air pressure from 100 to $200 \mathrm{~Pa}$ ) to prevent drag and heat loads on projectile surface during the strong acceleration phase (see Fig. 1). The allowed low pressure must be accurately determined to control the plasma phenomena on the armature and brushes.

The rocket projectile is embedded in a sabot casing. The sabot can be manufactured as light-weight sandwich structure based on CFRP/Aramid structure. During the acceleration process within the evacuated tube, this casing protects partly the projectile from mechanical and thermal loads. Mechanical loads are 
caused by the Lorentz force and the associated acceleration, friction with the ramp, guiding rods, and vibrations up to $1000 \mathrm{~Hz}$ due to the discontinuity of the current feeding different segments of the Distributed Energy storage System (DES). The friction provokes also the thermal loads on the sabot structure which are, however, manageable due to the short residence time in the tube. The nose part of the sabot container can additionally be reinforced and equipped with a honeycomb structure to withstand impact with the thin plastic membrane at the end of the ramp tube at high velocity. The function of plastic membrane is to seal the evacuated tube against air during the launch phase. Once the rocket launcher is released from the ramp, the sabot casing will be pyrotechnically separated. Due to the high initial velocity which is approximately $3.55 \mathrm{~km} / \mathrm{s}$ and the high aerodynamic drag of the sabot casing, its elements will separate and burn up.

Once the rocket launcher has passed the ramp, it will be sharply decelerated due to the atmospheric drag. Despite the enormous drag within the dense Earth atmosphere, the deceleration will be not higher than $100 \mathrm{~g}$. This is more than a factor of 30 lower than the acceleration on the launch ramp. The booster stage of the rocket vehicle will be ignited at an altitude of approximately $180 \mathrm{~km}$. At this altitude, the atmosphere is already rarefied and the launcher deceleration will be affected only by gravitation forces.

The most critical part of the LRA concept is the construction of the armature for the acceleration of the launch package. The armature can be of solid or plasma type. Solid armatures have high efficiency and are more reliable than plasma armatures. Unfortunately, they present a "velocity skin effect," i.e., at velocities on the order of $2 \mathrm{~km} / \mathrm{s}$, for many materials, the contact between armature and rails is damaged due to the evaporation of the armature material. This phenomenon is caused by the high concentration of current density at the rear side of the contact surface (plasma piston).

An alternative solution is to use a hybrid armature. It is basically a solid metal armature which completes the electrical contact with rails via plasma brushes. The application of plasma brushes, for the acceleration of masses in ranges of kilograms and more, is limited to velocities below $4400 \mathrm{~m} / \mathrm{s}$. At high sliding velocities the plasma brushes/bore erosion can be high and the loss of propulsive force will appear due to viscous drag caused by plasma and neutral gas ablation at tube walls. Gas ablation and ionization causes secondary arcs between rails and hypervelocity gouging on the rails. That is the reason to limit the initial velocity to $4400 \mathrm{~m} / \mathrm{s}$.

The power supply must be able to deliver very high current in a short period of time. The power of the acceleration scales with the mass. For example, the start of some launch packages with a mass of $650 \mathrm{~kg}$ and start velocity of $4.4 \mathrm{~kg} / \mathrm{s}$ requires the input electric power of $125 \mathrm{GW}$. Such power is beyond the current technical possibilities. This problem could be avoided due to temporal accumulation of energy. If the energy is assured from the commercial power 
network, first it has to be sucked at a low power, during intervals between two starts and short-term stored. During the launch phase, the accumulated energy must be released rapidly with the required high power. This approach reduces the power demands of the LRA system from the power network [8].

In the past, the batteries, capacitor banks, compulsators (high-speed compensated pulsed alternators), and homopolar generators were applied as power storage. The present states of art in this area are the capacitor banks, but they are expensive and have a short life circle (between 100 and 1000 discharging). As possible future solutions, discussed in the literature, fly wheels, superconductive magnetic energy storages (SMES) or even magnetohydrodynamic (MHD) generators could be envisaged. The maximum power to be applied for the LRA is primarily limited due the costs of a short-term energy storage source and the technical complexity of a power control/management system.

\section{ROCKET LAUNCHER FOR SMALL PAYLOADS}

Within preliminary design study, the properties of the entire rocket launcher, the propulsion system elements, and the proportions of the subsystems are considered. The proposed configuration with a cylindrical body and a conical flare is shown in Fig. 3. The vehicle has a total length of $6.2 \mathrm{~m}$ and total mass of $498 \mathrm{~kg}$. The aerodynamic shape enables reducing the drag and the heat loads. To obtain a low wave drag, the nose cone of the projectile has a spheri-

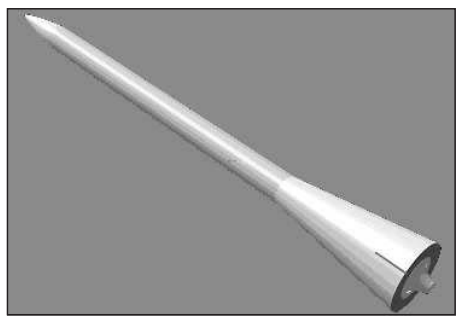

Figure 3 Proposed design of LEO payload carrier cally rounded tip with a 16 -millimeter radius and a contour designed according to the "power law" with an exponent $n=0.75$. The flare which is required to stabilize the launcher in flight has a length of $1.5 \mathrm{~m}$ and basis diameter of $0.6 \mathrm{~m}$. Due to its compact form (extremely important for LRA accelerators), a plug nozzle solution is selected. It additionally allows reducing the base drag in comparison to a Laval nozzle.

\subsection{Propulsion System}

As analyses show, the launch into LEO requires two stages (Fig. 4) which include one booster stage and one "kick-off" stage (Fig. 5). Each stage should be also equipped with a reaction control system (RCS) for trajectory corrections outside the dense atmosphere. DLR studies show that hybrid propulsion systems are the 


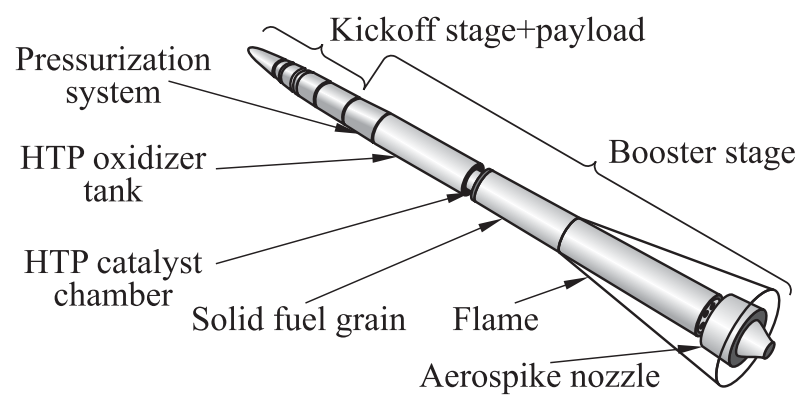

Figure 4 Sketch of the complete launcher with HRE of both stages

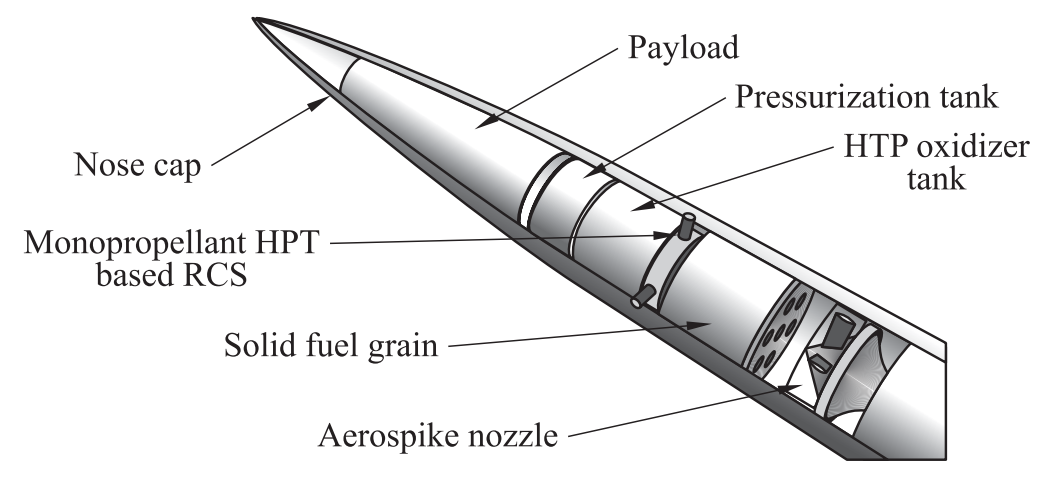

Figure 5 Payload container mounted on "kickoff" stage (based on HRE)

most promising solution for the investigated mission in comparison to liquid or solid propulsion. Their only drawback in comparison to liquid propulsion is the lower specific impulse.

As for the density impulse, the values for hybrid propellants are a bit lower or comparable with the solid propellants. This can be explained by the fact that the lower propellant density is compensated due the high specific impulse of advanced hybrid propellants. Advantages of HRE are safety, reliability, operating flexibility, simplicity, low development, and recurring costs, and low environmental impact.

However, there are some evident problems connected with HRE application, namely, keeping the oxidizer-to-fuel $(\mathrm{O} / \mathrm{F})$ ratio close to the operation point, a slightly lower grade of combustion (larger sliver fraction of solid grain), and lower combustion efficiency due to the change of the O/F ratio. Nevertheless, in the last years due to the intense investigations in the U.S., Europe, and Russia, a great number of experiments have been performed. The corresponding data 


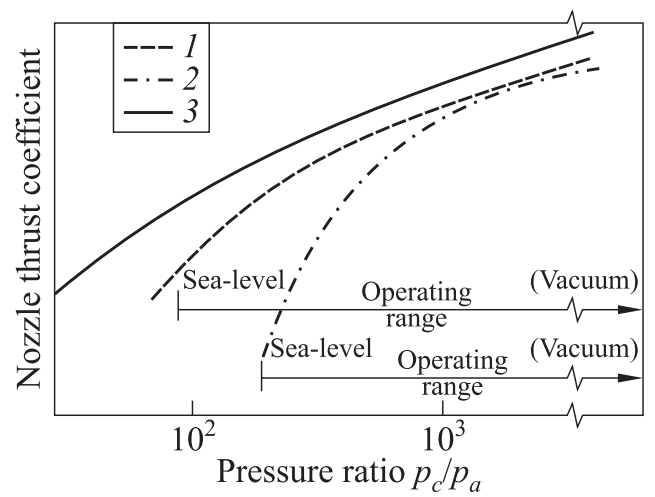

Figure 6 Performance comparison between aerospike (1) and bell-shaped (2) nozzles [10]; 3 - ideal nozzle (no losses)

emphasize that these disadvantages for hybrid propellants will be minimized and in short term the maturity of HRE could be established [9].

The presented analysis proposes the HRE concept which applies an advanced propellant combination based on HTP as oxidator and HTPB/Alane mixture as solid fuel. The proposed propellants for the HRE of a booster stage enable $I_{\mathrm{sp}}=320 \mathrm{~s}$ under vacuum condition and one similar mixture in the kickoff stage enables $I_{\mathrm{sp}}=343 \mathrm{~s}$. The corresponding HRE total impulse of booster stage is $1100 \mathrm{kN} \cdot \mathrm{s}$ and that of kickoff stages $44 \mathrm{kN} \cdot \mathrm{s}$.

The nozzle solution for a small launcher must be simple, compact, and effective. Nonextendable or actively-cooled nozzle is not desired. Such solutions have a high mass. Additionally, they are too complex and expensive. Another limit is the nozzle outlet diameter of $600 \mathrm{~mm}$ which is the consequence of the LRA accelerator ramp construction. The classic conical or bell-shaped nozzle has a very limited expansion ratio which leads to specific impulse losses. Also, the thrust coefficient loss could be $15 \%$ and more (Fig. 6) that is unacceptable [10].

An adequate solution can be a plug nozzle with truncated spike (aerospike). The ideal plug nozzle shows a long and heavy spike and, therefore, only truncated plug nozzles are of practical interest. Unlike a conical or bell-shaped nozzle, the plug nozzle flow is not fixed by the wall, but instead, the exhausted jet is bounded by external flow (Fig. 7). It is considered to have globally better performances because the jet boundary adjusts its shape to the ambient pressure and it expands optimal with varying altitude [11].

Disadvantages of plug nozzles are the more complex manufacturing and the necessity of active cooling if the engine burning time is longer than $30 \mathrm{~s}$. For short burning times in the range of 10 to $30 \mathrm{~s}$ (dependent of applied hybrid propellant), high thermal loads in area of critical nozzle throat are controllable without active 


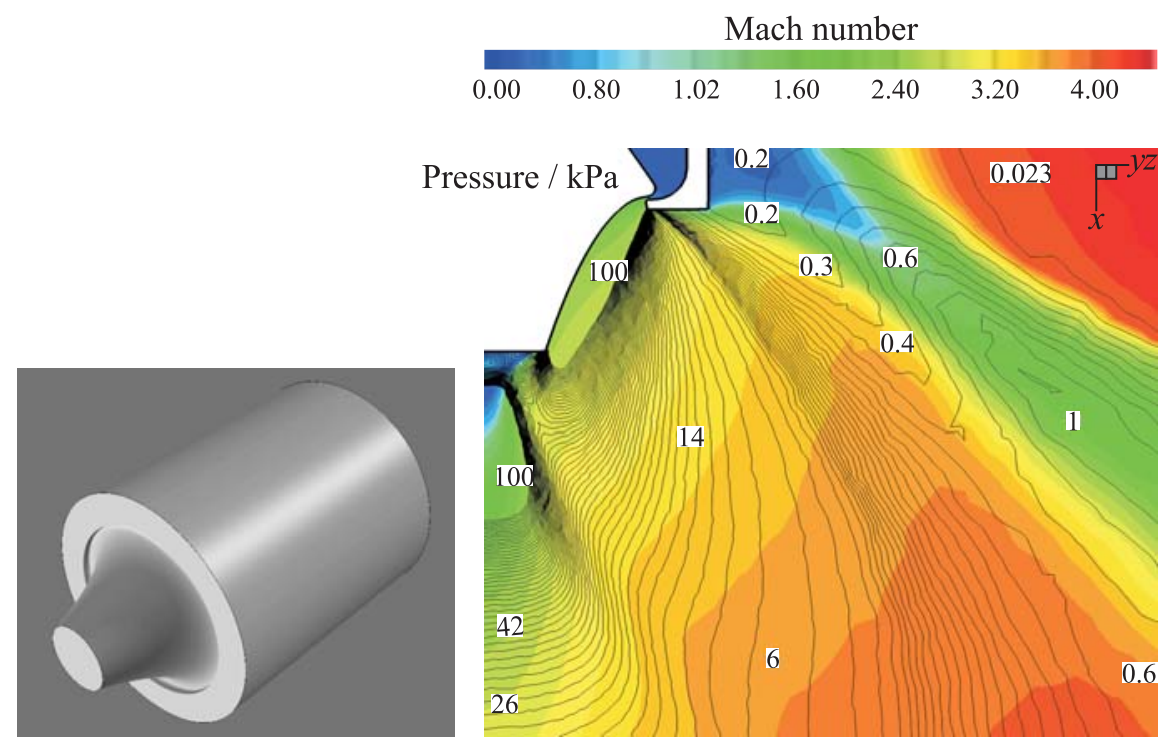

Figure 7 Flow simulation around plug nozzle at altitude $60 \mathrm{~km}$ (CFD simulation DLR Navier-Stokes code TAU [11]). (Refer Božić et al., p. 774.)

cooling. In this area, a reinforced thermal insulation based on passive cooling can be successfully applied. The detailed analysis to find an optimal start point of the booster engine on the projectile trajectory is under way, but early calculations indicate that it should be outside the dense atmosphere $(>80 \mathrm{~km})$. In this case, the gain of nozzle performances due to altitude compensation is not a primary criterion. More important are the compact shape and low nozzle mass. This importance is even higher for the "kickoff" engine which introduces the payload into LEO.

\subsection{Aerothermodynamics and Flight Mechanics}

\section{Thermal Loads to Projectile}

The direct launch of a vehicle into LEO starting from a LRA requires a launch velocity higher than $10 \mathrm{~km} / \mathrm{s}$. The direct launch means the possibility to reach the LEO only with LRA launch velocity without rocket propulsion. This velocity demand depends on the mission requirements, vehicle mass, and its aerodynamic properties. The total launch velocity is divided in: Earth orbital velocity which the payload should have to remain in desired orbit, gravitational losses during flight, and drag.

As shown in Fig. 8, due to turbulent effects and viscous drag at high Reynolds numbers, the nose of the projectile experiences very high heat loads and surface 


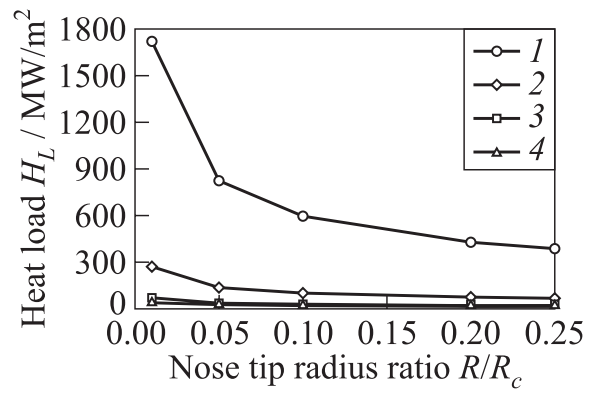

(a)

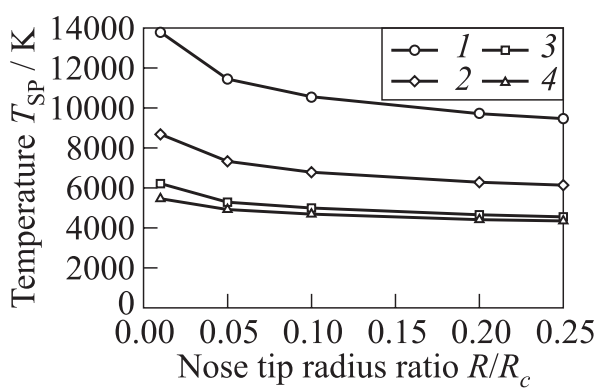

(b)

Figure 8 Heat load $(a)$ and surface temperature $(b)$ in SP vs. $R / R_{c}$ ratio for some selected Mach numbers: $1-\mathrm{Ma}=30 ; 2-18 ; 3-13$; and $4-\mathrm{Ma}=10.45$. Emissivity $\varepsilon=0.85$; altitude Alt $=200 \mathrm{~m}$; and $\mathrm{Pr}=0.7$

temperatures. The presented diagrams show heat loads (in $\mathrm{MW} / \mathrm{m}^{2}$ ) and surface temperatures $T_{W}$ (in $\mathrm{K}$ ) in the stagnation point of the vehicle, assuming steady flow conditions and air as a perfect gas. Based on the Fay-Riddell model, four characteristic Mach numbers of start from LRA (Ma $=30,18,13$, and 10.45) are compared.

The Fay-Riddell model predicts the heat transfer in the stagnation point of a blunt body based on the solution of the boundary layer equations [12]. The basic model formulation is derived for the equilibrium flow. The equilibrium flow implies infinite chemical and vibrational rates of air molecules. The applied model formulation is also valid for prefect gas, because the Lewis number for the ground condition is Le $=1$. In Fig. 8, the value marked Alt means that the selected start point of the LRA ramp end is $200 \mathrm{~m}$ above the sea level. For this point, the values of static pressure and density are insignificantly lower than at the sea level. So, the highest aerothermal loads are to be expected here. The heat loads and surface temperatures depend on the ratio of the nose tip radius $R$ and cylindrical body radius $R_{c}$. Taking, for example, a ratio of $R / R_{c}=0.1$ for the case with Ma $=30$ (corresponds to $V_{0}=10,209 \mathrm{~m} / \mathrm{s}$ ), which enables a visible reduction of aerothermal loads and acceptable rise of air drag [13], the aerothermal flux is approximately $H_{L, \mathrm{SP}}=600 \mathrm{MW} / \mathrm{m}^{2}$. This extremely high heat load is approximately a factor of 200 higher than usual heat loads during reentry from the Earth orbit. This case corresponds to a direct ground launch to LEO $[7,14]$ which is not feasible with the current and also near future technologies for thermal protection.

The case of starting from LRA with Mach number Ma $=18\left(V_{0}=6120 \mathrm{~m} / \mathrm{s}\right)$ corresponds to launching a small single-stage projectile with rocket assisted propulsion (kickoff engine). At $R / R_{c}=0.1$, the corresponding aerothermal load with $H_{L, \mathrm{SP}}=102 \mathrm{MW} / \mathrm{m}^{2}$ and $T_{W, \mathrm{SP}}=6786 \mathrm{~K}$ are difficult to manage. 
The possible technology based on intense and efficient ablation or active cooling for this purpose must be first developed. Also, the amount of mass for such thermal protection is relevant and as a first estimate shows that this TPS mass is higher than the LEO payload. Even for achieving the initial velocity of about $6 \mathrm{~km} / \mathrm{s}$, some known technological limitations by LRA development must be first solved $[6,15,16]$.

The analyzed case for a starting Mach number of Ma $=13$ (approximately $V_{0}=4400 \mathrm{~m} / \mathrm{s}$ - equal to scenario 2) corresponds to aerothermal loads which are still high but manageable using the present technology. Compared to typical reentry conditions, the heat loads are a factor of 6 to 10 higher but the exposition time is less than $30 \mathrm{~s}$. For example, at the stagnation point of the nose cap with $R / R_{c}=0.1$, the corresponding steady conditions for the launch point give $H_{L, \mathrm{SP}}=30 \mathrm{MW} / \mathrm{m}^{2}$ and $T_{W, \mathrm{SP}}=4996 \mathrm{~K}$. The reduction of the initial velocity below $4400 \mathrm{~m} / \mathrm{s}$ makes the LRA projectile design feasible today.

The launch from LRA with Ma $=10.45\left(V_{0}=3550 \mathrm{~m} / \mathrm{s}\right.$ - scenario 1) corresponds to the lowest reasonable limit for LRA length and represents the upper limit for the feasible projectile mass. At $R / R_{c}=0.1$, the corresponding aerothermal loads with $H_{L, \mathrm{SP}}=20.2 \mathrm{MW} / \mathrm{m}^{2}$ and $T_{W, \mathrm{SP}}=4600 \mathrm{~K}$ are relatively low and more common as compared to scenario 2 . Also, the projectile must be fabricated from advanced low-mass materials. An increase in the initial LRA velocity from 3550 to $4400 \mathrm{~m} / \mathrm{s}$ allows increasing the payload or (at constant payload mass) reaching higher the Earth orbits. Solutions for TPS in the last two cases $(\mathrm{Ma}=13$ and 10.45) are available today.

Under real flow conditions, due to the influence of real-gas effects (treatment of air as a real gas with inclusion of dissociation and catalysis), the aerothermal loads can be reduced by at least $10 \%$ compared to the calculated case with the Fay-Riddell model for a perfect gas. These conditions are realized for flight in the upper stratosphere. For the ground conditions, as in the calculated case, this gain is almost zero. The heat flux distribution along the front part of the rocket projectile, simulated for the initial trajectory point $(\mathrm{Ma}=12.96-$ scenario 2) with DLR Navier-Stokes code TAU, shows that it reduces rapidly with the distance from the nose tip and at a distance $L_{\mathrm{nc}} / D_{c}>2$ it can be handled with conventional TPS materials [6]. The TAU code simulation [17] is carried out for the perfect gas flow conditions and a radiation-adiabatic wall conditions with emissivity $\varepsilon=0.85$. For simulating turbulence, the SpalartAllmaras one-equation turbulence model is applied.

Three possible concepts are taken into account for cooling of the projectile nose tip during the ascent phase to LEO: passive cooling, transpiration cooling, and ablation cooling.

\section{Passive Cooling}

Figure 9 shows the temperature change with flight time at stagnation point of rocket projectile calculated for two different initial velocities (scenarios 1 and 2) 


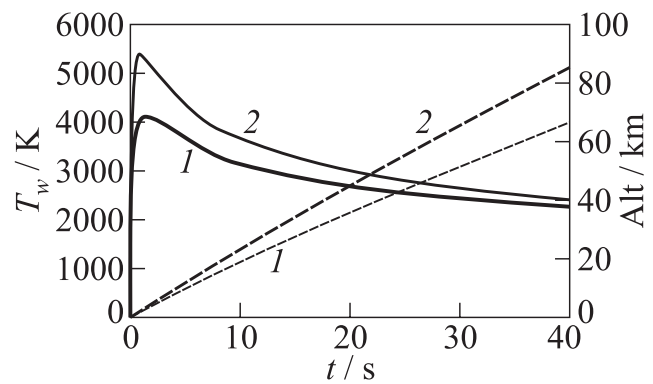

Figure 9 Temperature (solid curves) and altitude (dashed curves) change at SP of projectile with flight time for two different start velocities: $1-3550 \mathrm{~m} / \mathrm{s}$; and $2-$ $4400 \mathrm{~m} / \mathrm{s}$

with the Fay-Riddell model. The temperature in stagnation point $T_{W \text {,SP }}$ for angle of attack $\alpha=0^{\circ}$ (unconditionally stable flight) corresponds to the temperature on the top of the nose cap. The figure shows that the maximum surface temperature is reached within less than $1.5 \mathrm{~s}$ in both cases. Within this time, the interior structure temperature is lower than $T_{W, \mathrm{SP}}$. With further increase of flight time, $T_{W, \mathrm{SP}}$ decreases due to heat radiation from the surface to environment but still after $22 \mathrm{~s}$, the temperature is $T_{W, \mathrm{SP}}=2500 \mathrm{~K}$. In this point (for scenario 1), the projectile reaches the end of the dense atmosphere at $42 \mathrm{~km}$. For scenario 2 , the conditions are more critical - after $18.5 \mathrm{~s}$ of flight, the projectile achieves $42 \mathrm{~km}$ and temperature $T_{W, \mathrm{SP}}$ is still higher than $3150 \mathrm{~K}$. The interior structure temperature is certainly higher due to thermal resistance of structure material itself. This temperature in the vicinity of the nose tip is higher than the maximum application temperature for present TPS materials based on passive cooling. Despite the progress in the past (hafnia formers, UHTC, ULTRA 2000), passive cooling alone is not a satisfactory solution for LRA applications. Some promising TPS materials exhibit also too low compression strength to be used at high accelerations of about $3300 \mathrm{~g}$.

\section{Transpiration Cooling}

Second option is applying active cooling using a porous material for transpiration effect [18]. As materials for transpiration cooling, $\mathrm{Zr}_{2} \mathrm{O}, \mathrm{C} / \mathrm{SiC}$, or $\mathrm{Al}_{2} \mathrm{O}_{3} / \mathrm{SiO}_{2}$ mixtures are suitable. The cooling fluid flow through the porous material absorbs heat by convection and cools the material down. Under hypersonic flow conditions, inert gas (e.g., helium), gasified fuel (e.g., kerosene), or water is ejected at hot surface spots establishing a low-temperature sublayer within the boundary layer which overtakes a great part of surface heating. Liquid water shows the best cooling properties. If applied, water remains in liquid state during transpiration through the porous structure and remains an effective 


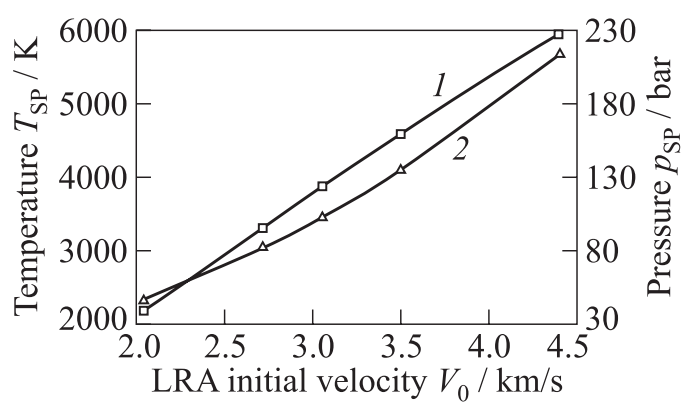

Figure 10 Wall temperature (1) and dynamic pressure (2) change at a stagnation point of the nose cap of projectile for characteristic Mach numbers at start point $\left(R / R_{c}=0.1\right)$; laminar flow; nose tip radius $R=16 \mathrm{~mm}$; emissivity coefficient $\varepsilon=0.8$; $\operatorname{Pr}=0.7$

cooling medium. Figure 10 shows the dependencies of the dynamic pressure and radiation adiabatic wall temperature in the stagnation point on the flight velocity for close-to-ground flight conditions. For the selected LRA initial velocities, the corresponding dynamic pressures in SP are $141 \mathrm{bar}\left(V_{0}=3550 \mathrm{~m} / \mathrm{s}\right)$ and $214 \operatorname{bar}\left(V_{0}=4400 \mathrm{~m} / \mathrm{s}\right)$. For such pressures, the saturation temperature of water steam is not achieved and it stays liquid (up to temperature 333.5 and $361.1^{\circ} \mathrm{C}$, respectively). For transpiration cooling feedthrough the interior, the pressure of the cooling medium must be higher than the stagnation pressure. A pressurized tank system for transpiration cooling based on water as a medium is possible but is not a rational solution. Preliminary analyses show that such a system is heavy. Its mass is higher than the mass of the payload.

\section{Ablations Cooling}

Today, ablative materials which satisfy the highest demands are available, e.g., carbon-carbon (C-Cs) monolithic braided ablative (MBA) or carbonphenolic (C-Ph) MBA. Carbon-carbons are very thermal-shock resistant and can be made in very stable geometries. The C-Cs can operate above $2000{ }^{\circ} \mathrm{C}$. During the ablation design, two parameters are of high importance: surface recession rate and back wall temperature $T_{\mathrm{BW}}$ of the heat shield. This temperature must be low to avoid damage of payload. If the $T_{\mathrm{BW}}$ is too large, further insulation is required which reduces the payload mass. Figure 11 shows the recession rate for $\mathrm{C}-\mathrm{Cs}$ as a function of stagnation pressure [19-21]. The recession rate should be less than $5.5 \mathrm{~mm} / \mathrm{s}$ for ground conditions with $V_{0}=4.4 \mathrm{~km} / \mathrm{s}$ (scenario 2) and less than $4.2 \mathrm{~mm} / \mathrm{s}$ for ground conditions with $V_{0}=3.55 \mathrm{~km} / \mathrm{s}$ (scenario 1). With increasing altitude the recession rate quickly decreases due to reduction of dynamic pressure and stagnation point temperature. In the considered case, the dense atmosphere is passed in $22 \mathrm{~s}$ and the ablation almost 


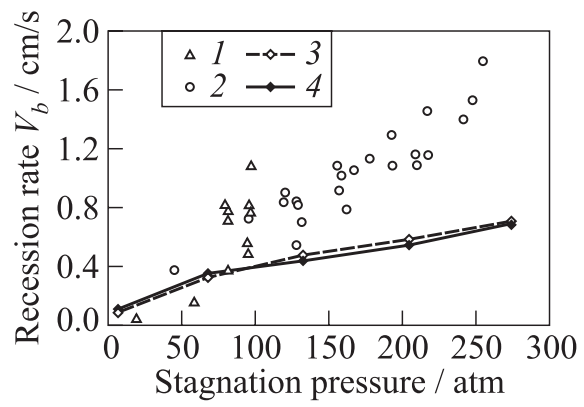

Figure 11 Recession rate as a function of stagnation pressure: 1 - 50-magawatt are data; 2 - ballistic range data; 3 - data of [19]; and $4-\mathrm{C}-\mathrm{C}$ ablation (data of $[19-21])$

disappears. It seems, after first analyses that only ablation is an acceptable solution for the aerothermal protection of the vehicle front part. If necessary, the transpiration cooling can be additionally used for effective cooling in the junction point between the cylindrical body and flare. The introduction of an additional cooling system in vehicle increases its mass and, therefore, the possible payload is reduced. The thermal stress in structure which appears during the flight of the propelled payload carrier is spatiotemporally displaced from those which appear in the ramp of the electromagnetic launcher. Until now, this problem is not explored and has to be understood prior to flight tests.

\section{Flight Mechanic Assessment of the Launcher}

The goal of the flight mechanic assessment is to confirm that the proposed Lorentz Rail accelerated launcher enables to reach approximately a $300 \times 400 \mathrm{~km}$ orbit. The study is performed applying a direct flight mechanic/aerodynamic coupling which is discussed in detail in [22]. As the aerodynamic results are based on the DLR surface inclination method SOSE, the aerodynamic results are calibrated by a comparison with selected TAU (Euler) calculations.

Figure 12 summarizes the representative results for $\mathrm{Ma}=5$. In view of the fact that the preliminary study is performed, the agreement between SOSE and TAU can be treated as very good. The aerodynamic layout of the launch vehicle takes into account that the complete configuration consisting of the both stages shown in Fig. 12 and the second (orbital) stage have to be statically stable. The first stage (booster) on its own has to be unstable to enable a save stage separation and re- entry.

The initial assumptions for the flight mechanic assessment are that the vehicle is launched at the San Marco Range (Italy) which is located at the east coast 


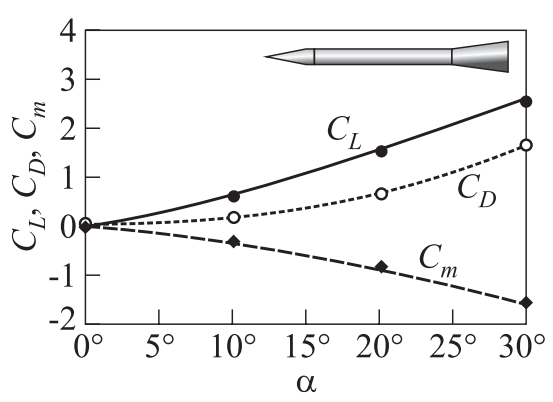

Figure 12 Calibration of the DLR surface inclination method SOSE (curves); symbols - TAU calculations; $\mathrm{Ma}=5$

of Kenya (latitude: $2.9^{\circ} \mathrm{S}$, longitude: $40.3^{\circ} \mathrm{E}$ ). It has to be pointed out that this location is chosen just in order to consider a realistic launch site. The initial flight path angle is $\gamma=39^{\circ}$, the initial velocity is $V_{0}=3550 \mathrm{~m} / \mathrm{s}$, the initial mass is $m_{0}=498 \mathrm{~kg}$ as introduced before. The vehicle is launched with a heading (azimuth) of $X=90^{\circ}$ to take full advantage of the Earth rotation. The calculations do not take into account any wind but the time dependence of mass, center of gravity $(\mathrm{CoG})$, and inertial tensor are included.

The first $50 \mathrm{~s}$ of the flight (6DOF) are illustrated in Fig. $13 a$. The dense part of the atmosphere (Alt $<42 \mathrm{~km}$ ) is passed after approximately $22 \mathrm{~s}$. After $50 \mathrm{~s}$, the vehicle is already at an altitude of $80 \mathrm{~km}$. The angle of attack oscillations indicate that the vehicle is dynamically unstable. Due to the quickly decreasing dynamic pressure, the pitch rate quickly decreases from $0.1 \% / \mathrm{s}$ until it is negligible after approximately $40 \mathrm{~s}$. During the first $50 \mathrm{~s}$ of the launch where the curvature of the trajectory is very small, the angle of attack is never higher than $\alpha= \pm 0.05^{\circ}$. With increasing flight time (Fig. $13 b$ ), it increases up to $\alpha \approx 3.4^{\circ}$ at $t=161 \mathrm{~s}$. Until this point, the increase of angle of attack is smaller than the change of the flight path angle. Therefore, the angle of attack increases for a configuration which flies without spin as is expected and additionally it underlines the sufficient static margin as the launcher stays almost aligned to the flight path. At $t=161 \mathrm{~s}$, the first stage is ignited. The thrust acts through the CoG along the body fixed longitudinal axis. This results in an increase of the flight path angle which, assuming that the attitude of the vehicle is constant, is responsible for the reduction of the angle of attack to $\alpha=0.8^{\circ}$ at $t=182.4 \mathrm{~s}$ (first stage burnout). At this time, stage separation is assumed and therefore, during the further flight, the second stage is almost aligned with the flight path although during the discussed assessment no RCS is taken into account.

The further flight (3DOF) and the injection into LEO is described in Fig. 14a. Due to the fact that the coupling procedure assumes no forces and moments at Alt $>122 \mathrm{~km}$ first both stages fly with an identical trajectory until the ignition 


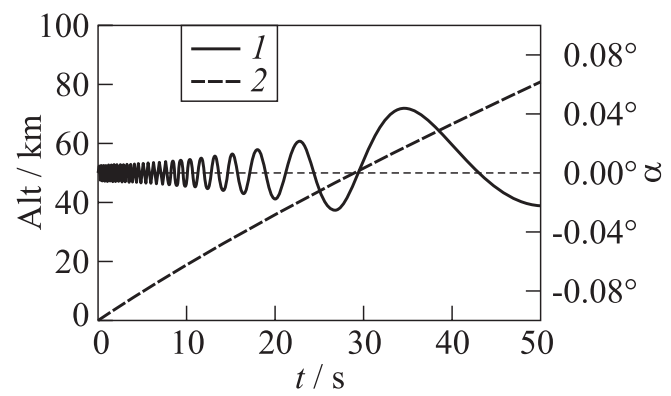

(a)

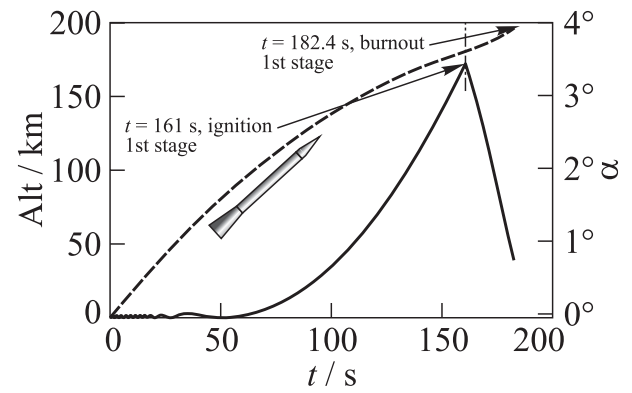

(b)

Figure 13 Launch phase of the trajectory, $V_{0}=3550 \mathrm{~m} / \mathrm{s}$ : dashed curves refer to Alt and solid curves to $\alpha$

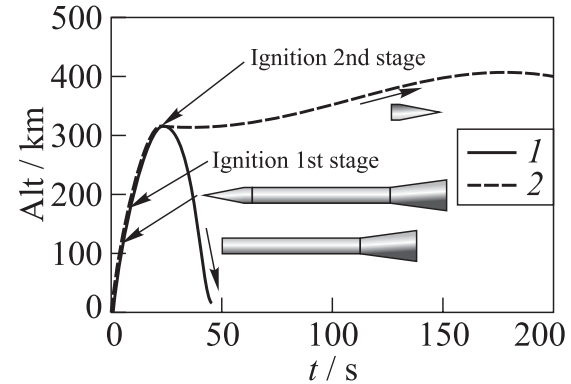

(a)

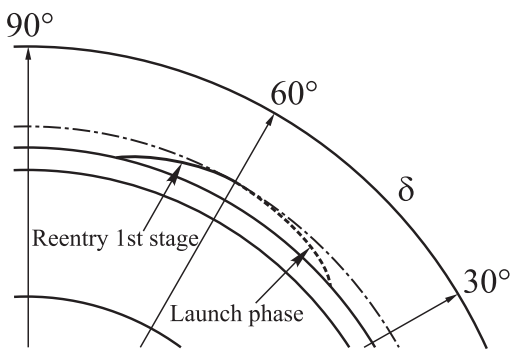

(b)

Figure 14 Illustration of the complete flight trajectory

of the 2 nd stage close before apogee $(t=450 \mathrm{~s})$. Then, the first stage reenters into the atmosphere whereas the second stage is boosted into the previously mentioned LEO (here: $340 \times 400 \mathrm{~km}$ ). Figure $14 b$ demonstrates an enlarged view of the launch and reentry phase in polar coordinates. It indicates that the 
first stage has a ground range of approximately $4000 \mathrm{~km}$ which has to be taken into account during the definition of a real launch mission.

In summary, the discussed flight mechanic assessment underlines the feasibility of the proposed Lorentz Rail accelerated launch vehicle. Even for an initial velocity of $V_{0}=3550 \mathrm{~m} / \mathrm{s}$, which represents the state of the art of Lorentz Rail technology, a $340 \times 400 \mathrm{~km}$ can be obtained almost without RCS which is not considered in the present study. An initial velocity of $V_{0}=4400 \mathrm{~km} / \mathrm{s}$ which takes into account the latest technology development of Lorentz Rail acceleration and material science enables much higher orbits.

\section{CONCLUDING REMARKS}

A preliminary design study of the LRA characteristics for the launch of nanosatellites into LEO is presented. Applied engineering methods allow determining the current needed to accelerate a rocket launcher, the length of the LRA ramp, and the input energy for launch package. The performance can be met by a LRA with the following characteristics: maximal current of about $6 \mathrm{MA}$, launcher length in the range 228-350 m, and maximal average acceleration on the order of $3300 \mathrm{~g}$. The input of electrical energy required for reaching these performances is in the range from 13.6 to 21 GJ. It has to be remarked that the entire LRA concept is in transition from Technology Readiness Levels TRL 2 to TRL 3 (NASA classification). For some components, such as the power supply, the upscaling of today's state-of-the-art (TRL 4) is fairly reasonable; other technological challenges require a step-wise approach. If the R\&D activities continue, within the next 8 years, TRL 6 could be achieved and within next 15 years, TRL 8 . This means that an actual system can be completed and "flight qualified" through test and demonstration.

Assuming an initial launch velocity of the LRA in the range from 3.55 to $4.4 \mathrm{~km} / \mathrm{s}$, the present analysis shows that a small staged projectile (full mass $498 \mathrm{~kg}$ ) propelled with a hybrid rocket engine, is capable of transporting $3 \mathrm{~kg}$ payload into LEO despite the harsh acceleration inside the LRA start ramp and the immense heat loads within the first $22 \mathrm{~s}$ of ascent flight. The selection of high technology materials with low density and high strength to withstand high accelerations up to $3300 \mathrm{~g}$ is one of the most important issues for further development of this advanced concept.

\section{REFERENCES}

1. Häfliger, G. 2006. Recherche Perspektiven Railgun — Kleinsatelliten und Wettbewerb. DLR internal Report. Nuglar, Schweiz. 
2. Fair H. D. 2003. Electromagnetic launch, IAT-UT. Int. J. Impact Eng. 29:247-62.

3. McNab, I. R., and F. C. Beach. 2007. Naval railguns. IEEE Trans. Magnetics 43(1, Part 2):463-68.

4. Lehmann, P., O. Božić, H. Grobusch, and J. Behrens. 2007. Electromagnetic railgun technology for the deployment of small sub-/orbital payloads. 7th Symposium (International) on Launcher Technologies. Barcelona, Spain.

5. Božić, O., J. M. A. Longo, P. Giese, and J. Behrens. 2006. High-end concept to launch micro-satellites into low-Earth-orbit based on combination of a two-stage rocket and railgun system. DGLR/STAB Symposium. Notes on Numerical Fluid Mechanics and Multidisciplinary Design. Berlin: Springer Verlag.

6. Božić, O. 2008. Possibilities of Lorentz electromagnetic accelerators for launch of payloads in low-Earth-orbit. 59th Astronautical Congress (International). Glasgow, Scotland, GB. Paper No. IAC-08-C.4.6.6.

7. Loeffler, M. J., and M. Schneider. 2009. High-speed plasma railguns: State of art and future. ISL Symposium on Pulsed Power Technology and Applications. Saint Louis, France.

8. Bérend, N., M. Calabro, O. Božić, C. Bonnal, G. L. Matloff, and D. Valentian. 2009. Non-chemical, non-nuclear advanced propulsion for space applications: Panorama and roadmap. 60th Astronautical Congress (International). Daejon, Korea. Paper No. IAC-09-C4.6.

9. Chiaverini, M. J. and K. K. Kuo, eds. 2007. Fundamentals of hybrid rocket combustion and propulsion. Progress in astronautics and aeronautics ser. Reston, Virginia: AIAA. 218.

10. Hutzel, D. K., and D.H. Huang. 1992. Modern engineering for design of liquidpropellant rocket engines. Progress in astronautics and aeronautics ser. AIAA, Washington: AIAA. 147.

11. Božić, O., and D. Porrmann. 2009. Estimation of flow field properties and thrust performances of axisymmetric plug - nozzles using CFD simulations. 6th Symposium (International) on Turbulence, Heat and Mass Transfer. Rome, Italy.

12. Anderson, J. D. 1989. Hypersonic and high temperature gas dynamics. New York: McGrow Hill Book Company.

13. Grozdzovskogo, G.L., ed. 1975. Aeromehanika sverhzvukovogo obtekanija tel vraschenija stepenoj formi. Moscow: Maschinostroenie. [In Russian.]

14. Milyayev, K., B. Urykov, and G. Webb. 2002. Some physical aspects of a direct launch of a payload into space. AAAF Symposium (International) Propulsion for Space Transportation in the XXI Century. Versailles, France.

15. Litrell, D. M., and K. A. Jamison. 1993. An experimental investigation of plasma armature railgun performance in the 3.2 to $4.2 \mathrm{~km} / \mathrm{s}$ regime. IEEE Transaction on Magnetics 29(1):859-64.

16. Avril, Ch., S. Hundertmark, and B. Reck. 2009. Composite material development for braking the $2.5 \mathrm{~km} / \mathrm{s}$ muzzle velocity barrier with the PEGASUS launcher. ISL Symposium on Pulsed Power Technology and Applications. Saint Louis, France.

17. Božić, O., T. Eggers, and D. Lancelle. 2010. Technological limitations for the design of Lorentz rail accelerated rockets. Symposium on Electrical Engineering. Saint Louise, France: French-German Institute. 
18. Van Foreest, A., M. Sippel, J. Klevanski, A. Guelhan, and B. Essser. 2007. Technical background and challenges of the space liner concept. 7th Symposium (International) on Launcher Technologies. Barcelona, Spain.

19. Keenan, J. A., and G. V. Candler. 1994. Simulation of graphite sublimation and oxidation under re-entry conditions. AIAA and ASME. 6th Joint Thermophysics and Heat Transfer Conference. Colorado Springs, CO: AIAA.

20. McNab, I. R., G. V. Candler, and C.S. Barbee. 2007. Projectile nose tip thermal management for railgun launch to space. IEEE Trans. Mag. 43(1):491-95.

21. Gosse, R., and G. Candler. 2007. Ablation modeling of electro-magnetic launched projectile for access to space. AIAA Paper No. 2007-1210.

22. Eggers, Th., and M. Gräßlin. 2008. Layout of hypersonic vehicles by coupling of aerodynamics and flight mechanics. 2nd International ARA Days on 10 YEARS AFTER ARD. Arcachon, Fance. 